\title{
Implicaciones de la conurbación en el sentido de comunidad y el apoyo social de la comunidad de Jongovito (San Juan de Pasto) ${ }^{2}$
}

Freddy Arley Ruano Bermúdez

Psicólogo

Universidad de Nariño, Colombia Correo electrónico: freddyarleyr@gmail.com

\section{Angela Jessica Quiroz Tepud \\ Psicóloga \\ Universidad de Nariño, Colombia \\ Correo electrónico: Jequiroz216@gmail.com}

\section{Jonnathan Harvey Narváez Burbano}

PhD. en Ciencias de la Educación

Universidad de Nariño, Colombia

Correo electrónico: jonnathanharnarvaez@gmail.com
Recibido: 05/09/2019

Evaluado: 01/10/2019

Aceptado: 05/11/2019

\section{Resumen}

El acelerado crecimiento de las ciudades en Latinoamérica ha permitido el desarrollo de fenómenos sociales como la conurbación, donde las nuevas dinámicas afectan la salud mental de los habitantes; de esta manera, el objetivo es analizar las implicaciones de la conurbación en el sentido de comunidad y el apoyo social. El estudio se desarrolló a través de la metodología cualitativa, el enfoque histórico hermenéutico y el tipo de estudio microetnografía, usando las técnicas observación participante, grupo focal y mapa parlante. Los resultados evidencian que existe una afectación en los componentes, expresados en la pérdida de valores e identidad, las dificultades en las relaciones sociales, problemas de salud pública y redes de apoyo, permitiendo analizar a profundidad las transformaciones psicosociales que han experimentado los habitantes de la comunidad a raíz del crecimiento urbano y la importancia que representan los factores psicosociales como determinantes de la salud mental comunitaria.

Palabras clave

Salud mental, conurbación, sentido de comunidad, apoyo social, comunidad.

2 Para citar este artículo: Ruano, F., Quiroz, J. y Narváez, J. (2021). Implicaciones de la conurbación en el sentido de comunidad y el apoyo social de la comunidad de Jongovito (San Juan de Pasto). Informes Psicológicos, 21(2), pp. 27-42 http://dx.doi.org/10.18566/infpsic.v21n2a02 


\section{Implications of the conurbation in the sense of community and the social support of the community of Jongovito (San Juan de Pasto)}

Abstract

The accelerated growth of cities in Latin America has allowed the development of social phenomena such as the conurbation, where the new dynamics affect the mental health of the inhabitants. In this way, the objective is to analyze the implications of the conurbation in the sense of community and social support. The research was developed through qualitative methodology and was also based on the hermeneutical historical approach and the microethnography type of study. Participant observation, focus group and talking map techniques were applied. The results show that there is an affectation in the components, expressed in the loss of values and identity, difficulties in social relationships, public health problems and support networks, allowing an in-depth analysis of the psychosocial transformations that the inhabitants of the community have experienced, as a result of urban growth and the importance of psychosocial factors as determinants of community mental health.

Keywords Mental health, conurbation, sense of community, social support, community.

\section{Implicações da conurbação no sentido de comunidade e apoio social da comunidade de Jongovito (San Juan de Pasto)}

Resumo

0 crescimento acelerado das cidades na América Latina tem permitido o desenvolvimento de fenômenos sociais como a conurbação, onde as novas dinâmicas afetam a saúde mental dos habitantes. Desta forma, o objetivo é analisar as implicações da conurbação no sentido de comunidade e apoio social. 0 estudo foi desenvolvido por meio de metodologia qualitativa, abordagem histórica hermenêutica e estudo do tipo microetnografia, utilizando técnicas de observação participante, grupo focal e mapa falante. Os resultados mostram que existe uma afetação nos componentes, expressa na perda de valores e identidade, dificuldades nas relações sociais, problemas de saúde pública e redes de apoio. Estes resultados permitem então uma análise aprofundada das transformações psicossociais que os habitantes da comunidade experimentaram como resultado do crescimento urbano, e da importância dos fatores psicossociais como determinantes da saúde mental da comunidade.

Palavras chave Saúde mental, conurbação, senso de comunidade, suporte social, comunidade. 


\section{ntroducción}

En las últimas décadas el desarrollo social y el crecimiento demográfico en Latinoamérica se han incrementado de manera exponencial, como consecuencia de los procesos de urbanización y las dinámicas de adaptación que experimentan las poblaciones; este crecimiento nace de las transformaciones productivas y sociales vinculadas con las estrategias globales de industrialización, las cuales promueven una serie de habilidades económicas que favorecen la modernización y el crecimiento de las ciudades frente a sus periferias (Da Cunha \& Vignoli, 2009). En Colombia el desarrollo de la urbanización y el crecimiento demográfico presentan las mismas características, puesto que a menudo se desarrollan procesos configuracionales, donde las periferias evolucionan en tiempo y espacio, con el fin de hacer frente a las nuevas necesidades económicas y sociales, las cuales se encuentran enmarcadas en un proceso denominado conurbación (Martínez, 2015).

Moreno (2008) acuña el término conurbación como un fenómeno en el cual dos o más ciudades o poblaciones se integran dando lugar a una nueva territorialidad. Además, comprende la relación entre los factores sociales, culturales, políticos y económicos; no obstante, y a pesar de su estudio en 1915, existe escasa literatura que analiza las implicaciones sociales y culturales que representa la unión de los territorios (Bedoya \& Muñoz, 2013). Por ejemplo, Chaparro (1972) relaciona el fenómeno de conurbación con los cambios socioculturales y comunitarios que experimentan las zonas rurales ubicadas en la periferia de la ciudad.

Dichos cambios se expresan en función de las necesidades psicosociales que experimentan los miembros de una comunidad o poblaciones que terminan formando parte de una estructura unificada, donde las dinámicas de la ciudad se instauran en los procesos sociales propios de las comunidades aledañas y que, a menudo, se evidencian en la disminución o afectación negativa de los sentimientos de pertenencia, cohesión, integración y conexión emocional (Chamorro, 2013; McMillan \& Chavis, 1986) así como también en la percepción de apoyo social o recursos psicológicos, que brindan o reciben para poder enfrentar los nuevos retos, los cuales son predictores del desajuste comunitario y que a menudo se constituye como el punto de análisis de problemas de salud comunitaria (Fachado, Menéndez \& González, 2013), como el consumo de sustancias psicoactivas, embarazos en adolescentes, deserción estudiantil, pobreza, pérdida de identidad, violencia y delincuencia.

Por consiguiente, el análisis dimensional del sentido de comunidad y el apoyo social como procesos que forman parte de la estructura comunitaria (Ruano, Hernández \& Dorado, 2019), se pueden considerar como elementos indispensables en la comprensión, organización, funcionamiento y evolución de los grupos sociales y/o comunidades (Maya, 2014).

Por su parte, en la ciudad de San Juan de Pasto los procesos de conurbación se desarrollan de forma similar al de las grandes ciudades de Colombia, debido a que la expansión incontrolada permite la ocupación de espacios que, en su mayoría, 
no se encuentran en condiciones adecuadas para su habitación, así como también el crecimiento de la zona urbana acarrea problemáticas como las necesidades en la prestación y mejoramiento de los servicios públicos (Rojas \& Viveros, 2016). En el caso del corregimiento de Jongovito, comunidad perteneciente a la comuna 6 del Municipio de San Juan de Pasto, el crecimiento de la ciudad hacia sus territorios no solo ha disminuido la distancia entre los territorios, sino que, además, se evidencian procesos de conurbación, por cuanto sus habitantes perciben amenazante en su estructura social el influjo de los imaginarios y prácticas de la ciudad que se sobreponen a las dinámicas históricas de la comunidad.

A menudo sus habitantes perciben como consecuencias del cambio, el consumo de sustancias psicoactivas en adolescentes, deserción escolar, delincuencia, la reducida participación de la comunidad en procesos comunales, la pérdida de la tradición oral en festividades propias, el rescate de la memoria colectiva y el desinterés en el desarrollo de prácticas agrícolas tradicionales. Es por eso que se hace pertinente analizar en un principio el sentido de comunidad de los habitantes del corregimiento, lo cual implica orientar el análisis desde los componentes del sentido y, en segundo lugar, identificar las fuentes de apoyo social, así como también sus niveles de estudio para promover una comprensión del fenómeno desde lo teórico y que se fortalezca la apropiación de los significados dentro del proceso de construcción social de la comunidad cuya historia data alrededor de 15 años (Burbano, Narváez \& Palacios, 2017).

\section{Componentes del sentido de comunidad}

Desde los aportes de McMillan y Chavis (1986) se comprende que el sentido de comunidad es la percepción que tiene un individuo o grupo social de pertenecer y responder de forma integral hacia una estructura mayor, es un factor multidimensional, es decir, que se encuentra determinado a partir de cuatro elementos que interactúan en los diferentes escenarios donde las relaciones humanas consolidan la experiencia comunitaria (Montero, 2004). El primero de ellos es la membresía, vista como el sentimiento de pertenencia hacía una determinada red o vínculo de relaciones sociales y que, a su vez, permite diferenciar a los miembros que hacen parte de la comunidad a través de elementos como límites geográficos y simbólicos, seguridad emocional y confianza y la satisfacción de las necesidades, sentimientos de pertenencia e identificación e historia de acuerdo a los hechos o acontecimientos vivenciados. El segundo es la influencia recíproca determinada como la influencia bidireccional entre las acciones de la comunidad y las del individuo, es decir, que la percepción que se tiene del poder dentro del colectivo, comprueba su grado de atracción hacia el mismo.

En tercer lugar, la integración y satisfacción de necesidades comprendidas como las metas colectivas y que, al mismo tiempo, son priorizadas. En cuarto lugar, la conexión emocional, donde los lazos afectivos entre el individuo y la comunidad, evidenciados en la búsqueda conjunta de alternativas ante un problema aumentando la creación de redes y el apoyo social entre los miembros 
(MacMillan \& Chavis, 1986, citados por Cueto, Espinoza, Guillen \& Seminario, 2016).

\section{Dimensiones del apoyo social}

Bowling (1991) define el apoyo social como un proceso relacional en el que el ser humano consigue diferentes tipos de ayuda, ya sea emocional, instrumental o económica de su red vincular comunitaria y que promueve no solo el bienestar y el cooperativismo (Cerquera, Matajira, Salas \& Uribe, 2018), sino que, además, permite el ajuste psicosocial y mejora la cohesión grupal (Bonilla, Panadero, Rivas, Vásquez \& Vásquez, 2017). Este concepto se encuentra clasificado en 4 dimensiones: la primera es el apoyo informativo, es decir, todo tipo de información transmitida con el fin de enfrentar las problemáticas y ayudarse así mismo. La segunda es el apoyo instrumental, vista como una serie de ayudas materiales que favorecen directamente a la persona que lo necesita. La tercera es el apoyo efectivo, comprendido como expresiones de afecto y amor empleadas por los miembros de la comunidad. La cuarta es la interacción social positiva, donde prima el tiempo que se comparte entre los habitantes, capacidad de distracción o disponibilidad.

De igual manera, en el apoyo social es importante reconocer las fuentes que propone House (1981), en las que se encuentran: el conyugue/compañero, familiares, amigos, vecinos, jefes o supervisores, compañeros de trabajo, personas de servicio o cuidadores, grupos de autoayuda y profesionales de la salud o servicios sociales (Fachado et al., 2013). Teniendo en cuenta el marco explicativo que analiza y justifica el estudio, el presente artículo de investigación tiene como objetivo analizar las implicaciones de la conurbación en el sentido de comunidad y el apoyo social dentro de la comunidad de Jongovito.

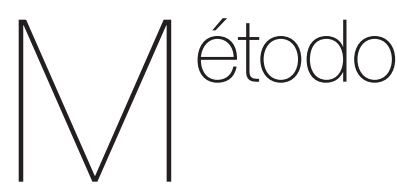

El presente artículo se desarrolla a través del paradigma cualitativo enfocándose en la comprensión de las realidades de los habitantes (Quintana \& Montgomery, 2006). El enfoque es el histórico hermenéutico, ya que se construye un campo de reflexión sobre la interpretación del sentir y pensar de la comunidad, permitiendo la interacción como resultado de la experiencia de los habitantes de la comunidad (Gómez \& Villalobos, 2014). El tipo de estudio es la microetnografía, herramienta metodológica en la que se realiza un recorrido y, por consiguiente, se describen rigurosamente los cambios sociales y físicos que ha experimentado la comunidad (Buriticá, Chaverra \& Monsalve, 2015; Fardella, Jiménez \& Muñoz, 2017).

\section{Participantes / Unidad de análisis}

Se contó con la participación de 20 líderes comunitarios del corregimiento de Jongovito, entre 30 y 50 años, de género masculino y femenino. Como criterios 
de inclusión se establecieron: 1) Haber nacido en el corregimiento; 2) Vivenciar o experimentar los efectos de la conurbación en los últimos 20 años; y 3) Ser líder o lideresa comunitario. Como criterios de exclusión se constituyeron: 1) No pertenecer a la vereda Jongovito Centro y 2) Ser menor de edad.

\section{Técnicas e instrumentos}

- Observación participante, la cual permitió realizar un análisis profundo del discurso y las formas o expresiones lingüísticas de los participantes (Jociles, 2018). Además, fue desarrollada con los adultos mayores, dado su experiencia y conocimiento comunitario. Las dimensiones trabajadas fueron las relacionadas con sentido de comunidad y apoyo social y, finalmente, sistematizada a través del diario de campo, durante el reconocimiento de la comunidad en cada una de las sesiones.

- Grupo focal, donde se valora la interacción social entre el investigador y el grupo, que, además de profundizar sobre los aspectos planteados, permite reconocer los sentires y comportamientos propios de los miembros en un contexto determinado (Buss, Coelho, De Oliveira, López \& Mikla, 2013). Esta técnica se llevó a cabo con 18 miembros de la comunidad en 3 diferentes sesiones organizadas de acuerdo a los aspectos a evaluar. Inicialmente se discutió sobre el estado general de la comunidad, seguido de las dimensiones del sentido de comunidad y, por último, las dimensiones del apoyo social en las que se procuró propiciar un espacio de opinión y discusión para captar las experiencias de los habitantes y obtener aproximaciones cualitativas.

- Mapa Parlante, técnica que permite organizar y comunicar decisiones o reflexiones de la comunidad a través de la representación gráfica de mapas territoriales (Suárez, 2015). Además, se aplicó con 6 participantes y facilitó la representación gráfica de los cambios experimentados en materia de ubicación geofísica, el nivel de crecimiento de la ciudad, las zonas afectadas por el crecimiento demográfico y el impacto ambiental que vive la comunidad.

\section{Procedimiento}

El procedimiento fue seleccionado de acuerdo a las necesidades del proceso y atendiendo a las consideraciones planteadas por la microetnografía como una herramienta metodológica que permite la descripción, interpretación, comprensión y difusión del contexto analizado rigurosamente; las etapas se formularon de acuerdo a los postulados de Fardella et al. (2017).

\section{Etapa: 1. Familiarización:}

A partir del proceso desarrollado en la investigación se realizó un acercamiento previo a los líderes y lideresas comunitarios, quienes apoyaron la iniciativa investigativa y facilitaron el reconocimiento de las condiciones físicas y sociales del corregimiento. 


\section{Etapa 2. Recolección de información:}

Inicialmente se realizó el proceso de selección de los actores que conformarían la unidad de análisis; seguidamente, a través de estrategias de convocatoria como chapolas, invitaciones personalizadas y anuncios en la oficina corregimental, se logró citar a la población a los encuentros, los cuales estuvieron organizados teniendo en cuenta el cronograma de actividades mensuales. Los grupos fueron conformados en función del diseño de cada grupo focal y además asociados a las categorías de sentido de comunidad, así como también en el desarrollo del mapa parlante, el cual permitió dinamizar la organización de los encuentros. Por último, para poder fortalecer el proceso investigativo de las dimensiones de apoyo social se generó el diario de campo. Además, se contó con el aval de expertos en el campo, quienes mensualmente evaluaron, corrigieron y dieron el aval de cada técnica para continuar con su respectiva ejecución.

\section{Etapa 3. Análisis de la información:}

De acuerdo con Álvarez (2008) el análisis de la información dentro del proceso microetnografico se desarrolla mediante la interpretación de significados y el análisis de las dinámicas culturales propias de la comunidad. En este sentido, como primer paso se realizó un análisis crítico de los datos, donde además de transcribir y organizar los resultados por cada una de las técnicas e instrumentos empleados se reflexiona sobre lo vivido o experimentado. Como segundo paso se hizo una selección y reducción de datos de acuerdo a los intereses del estudio. Como tercer paso se organizaron y categorizaron los datos de forma manual, con el fin de darle sentido a la información recolectada y establecer las relaciones entre las categorías que, si bien estaban determinadas por los elementos teóricos, estaban sujetas a diferentes interpretaciones. Como quinto paso, y para garantizar el enfoque integral, los análisis fueron sometidos a diferentes evaluaciones por parte de los miembros de la comunidad, los cuales realizaron retroalimentaciones significativas al proceso.

\section{Conflicto de Intereses}

No se presentó ningún conflicto de intereses por parte del equipo de investigación.

\section{Consideraciones Eticas}

El proceso investigativo tuvo como precedente establecer una serie de acuerdos e indicaciones que permitan salvaguardar los principios de la Ley 1090 de 2006, en la que es necesario proteger la identidad de los participantes; también fue importante reconocer que el manejo de la información propende por respetar los principios de libertad, no maleficencia y autonomía de la población en estudio. De igual manera, la iniciativa respalda las funciones de la Universidad de Nariño, en las que se resalta que los resultados de las investigaciones deben promover la transformación y la interacción social. Para ello, a través de la Vicerrectoría de Investigaciones, se contó con el aval administrativo para la ejecución del proyecto y, por consiguiente, los productos. 
Durante cada fase del proceso los participantes reconocieron lo siguiente: 1) que el estudio no representó ningún riesgo; 2) cada participante tenía el derecho de participar voluntariamente de las sesiones; 3) que formaran parte en el proceso de evaluación del estudio; 4) como herramienta de apoyo los investigadores podrían utilizar medios audiovisuales, especialmente grabadora de audio para registrar algunas sesiones y que no representaría ningún riesgo o divulgación; y 5) para legitimar estas consideraciones se aprobarían a partir del diligenciamiento del consentimiento informado.

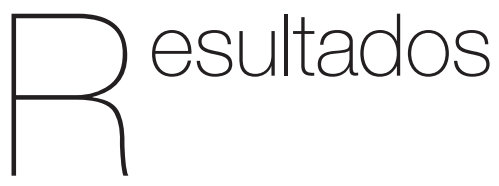

Los resultados de la investigación se presentan teniendo en cuenta el análisis de las implicaciones de los componentes del sentido de comunidad y el apoyo social, mientras que las expresiones que se organizan entre comillas hacen referencia a los relatos de los habitantes, los cuales reflejan la influencia de los efectos de la conurbación.

\section{Membresía}

La investigación evidenció que las transformaciones en los sentimientos de pertenencia hacia la red de apoyo se expresan por medio de los cambios en los sistemas de valores comunitarios característicos de los habitantes, pues existe una tendencia hacia el individualismo, la apatía y la ausencia de apoyo, los cuales se analizan como una adopción de dinámicas sociales propias de la ciudad. Además, la disminución de sentimientos de identificación histórica, la baja participación en actividades culturales y tradicionales favorece la ausencia del conocimiento sociohistórico, mientras que el aumento en el uso de tecnologías de la comunicación afecta desfavorablemente la interacción social entre grupos etarios. Entre las expresiones más comunes se pueden considerar las siguientes:

"Cuando se fundó nuestro
corregimiento, los adultos mayores
estábamos organizados muy bien,
éramos solidarios entre nosotros
mismos, nos gustaba apoyarnos y
cualquier problema se solucionaba
de buena manera, ahora en
Jongovito cada uno cuida de sí
mismo, esa fraternidad de vecinos
se ha perdido".

De igual manera, la disminución de los límites geográficos entre la ciudad y el corregimiento incrementa el espacio físico y la demografía del corregimiento provocando la reducción de zonas límites entre corregimientos, que como consecuencia acarrea problemáticas ambientales y de seguridad. Los siguientes relatos son algunas expresiones:

"Hemos notado que así como Pasto se creció, nuestro corregimiento también, antes era una veredita, ahora tiene mucha gente que uno ni conoce".

\section{Influencia recíproca}

Las diferencias en las percepciones del poder se consolidan como un 
elemento causal de la baja participación comunitaria, pues la mayoría de los habitantes desconocen los procesos organizacionales que desarrolla la comunidad y producto del desinterés y el malestar social es la concentración del poder en algunos miembros, por lo que los procesos democráticos no se desarrollan de manera efectiva. En la siguiente expresión evidencia esta transformación:

"Uno como líder comunitario 10 que busca es el bien para todos, no importa de dónde venga ni qué haga, pero a veces las personas creen que uno no hace nada...".

Otro producto de la influencia recíproca está en el aumento de factores de riesgo en salud mental; en este sentido, el consumo y el expendio de sustancias psicoactivas y la delincuencia son el resultado, según los habitantes, de las dinámicas de interacción de los jóvenes de la ciudad y los de la comunidad, prácticas que en la antigüedad no se presentaban. Además, el uso excesivo de tecnologías de la comunicación como redes sociales y la televisión permite desarrollar percepciones negativas de los adultos mayores hacia los cambios en las actitudes de jóvenes. En este caso, se determina el fundamento:

"Nuestras juventudes eran muy diferentes mucho antes de que el corregimiento avanzara, eran muy participativos, solidarios, no había jóvenes consumiendo drogas, pero como los males de la ciudad han llegado aquí, los jóvenes de la ciudad corrompen a los nuestros, los envician, los llevan por el mal camino".

\section{Integración y satisfacción de necesidades}

Los cambios en las metas colectivas corresponden a diferentes factores: por un lado se encuentran las diferencias generacionales, las necesidades y los retos que viven los adultos mayores, quienes asocian sus necesidades con la recuperación del conocimiento o saber histórico y, por otro lado, la pérdida de tradiciones con relación al intercambio de recursos agrícolas se ha incrementado debido a la falta de oportunidades para los miembros, ya que las principales fuentes de empleabilidad se relacionaban con el trabajo en las "ladrilleras". Evidencia de esto, se puede encontrar la siguiente afirmación:

\begin{abstract}
"Antes teníamos la tradición de intercambiar productos agrícolas que nosotros mismos cultivábamos, papa, maiz, frijol o las cebollas nunca faltaban, pero con la construcción de ladrilleras la tierra dejó de ser fértil y ya no se siembra nada".
\end{abstract}

Asimismo, la explotación de los recursos naturales como la tierra ha generado impactos en el medio ambiente y en la salud, pues la tierra ya no posee las mismas propiedades minerales que antes y los efectos del humo de las ladrilleras se ha convertido en una causa de afecciones respiratorias en los niños y niñas. En este sentido, la siguiente expresión da cuenta del análisis:

"Lo poco que cosechamos es para uso mismo de la casa, muchos tienen que ir hasta la ciudad a comprar las cosas que solíamos tener, el daño a nuestra tierra es muy visible y nadie hace nada, las 
ladrilleras cada vez hacen más daño a nuestros niños, los enferma, ese olor a quemado es en todo el corregimiento".

\section{Conexión emocional compartida}

Los sentimientos de desvalorización hacia los adultos mayores se han incrementado tras el aumento de la cultura moderna y la migración de las familias tradicionales; en este sentido, los espacios para compartir en familia han disminuido por el aumento de las tecnologías de la comunicación y el desinterés por compartir experiencias en familia.

Además, la pérdida de los valores comunitarios favorece el crecimiento de problemáticas relacionadas especialmente la exclusión social que experimentan los adultos mayores, reduciendo sus posibilidades de sentirse valorados por su esfuerzo, compromiso y trayectoria en la comunidad. A continuación se presentan los siguientes relatos como fuentes de análisis:

"Las relaciones han cambiado, los adultos no son valorados, ni se los tiene en cuenta, ellos son, como dicen, un estorbo, en esta sociedad tan materialista"; "La familia era lo más sagrado, era bonito ver a todos en la misa o en el parquecito".

Además del impacto que se presenta en las dimensiones del sentido de comunidad, afecta las percepciones y sentimientos que caracterizan el apoyo social entre los habitantes; por lo tanto, a continuación se presenta la descripción de las transformaciones observadas y analizadas en la técnica del diario de campo.

\section{Apoyo social informativo}

Se evidencia que existe diversidad en los sistemas informativos y estrategias de comunicación por parte de la comunidad. Estos han perdurado en los últimos años, sin embargo, la satisfacción respecto a estos no es suficiente para cubrir las demandas del contexto, especialmente en lo que respecta a problemáticas de inseguridad como robos o accidentes y también de violencia como riñas o violencia intrafamiliar, puesto que la red de seguridad de la comunidad es ineficaz por el desconocimiento de los miembros respecto a las rutas de gestión en seguridad, mientras que los programas de formación de líderes en seguridad, una estrategia de convivencia de la Alcaldía y la Policía, no presenta resultados eficaces por la ausencia de material de apoyo y formación ciudadana.

De igual manera, es importante reconocer que dentro del corregimiento no existe una estación de policía que favorezca el apoyo a los habitantes. Por otro lado, no hay una vinculación efectiva con el puesto de salud, pues según los participantes éste no opera de acuerdo a las necesidades de los pobladores.

\section{Apoyo social instrumental}

Se presenta una disminución significativa de las fuentes de apoyo, especialmente la comunitaria, ya que las percepciones de desconfianza se han incrementado con el aumento de problemáticas de inseguridad y delincuencia. Asimismo, se evidencia una tendencia hacia el individualismo por parte de 
los habitantes, pues el desinterés en los procesos comunitarios es producto de la centralización de las estructuras de poder y de los recursos, especialmente en sectores como la cabecera corregimental, permitiendo la división entre miembros de la comunidad.

\section{Apoyo social efectivo}

La disminución de las fuentes de apoyo familiares es un efecto más de la conurbación pues la comunidad determina que el uso desmedido de herramientas de comunicación promueve la desintegración entre las familias; además, la primacía de las necesidades económicas sobre el bienestar social evidencia no solo la reducción de las relaciones entre padres, hijos y abuelos sino que da cuenta de problemáticas de salud pública como la violencia intrafamiliar, la violencia de género y las necesidades económicas a las que se enfrentan muchas familias del sector.

\section{Interacción social positiva}

A partir de la transformación en los espacios de interacción social experimentados por los miembros, es importante analizar la apatía hacia la participación de actividades tradicionales y programas institucionales, pues en ellos se puede evidenciar la pérdida de intereses comunitarios y de cohesión social de la comunidad, como consecuencia de las diferencias entre los sectores o miembros, por ejemplo, los jóvenes consideran que una fuente de apoyo que facilita su comunicación son los sistemas virtuales.

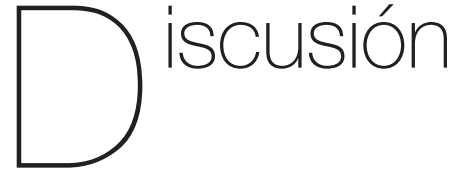

A partir de los resultados encontrados se establece que las implicaciones sociales del fenómeno de conurbación en los habitantes son evidentes, en el sentido de que el crecimiento de la ciudad y la absorción del corregimiento configura los sistemas tradicionales y los procesos comunitarios (Moreno, 2008). De esta manera, la expansión de la ciudad de San Juan de Pasto transforma la identidad de la comunidad y aumenta las problemáticas de carácter personal, familiar, social, organizativo y ambiental, elementos constitutivos de la salud mental comunitaria, pues estos factores determinan el desarrollo adecuado de entornos que generan bienestar psicosocial a los miembros ya que, de acuerdo con Martínez (2015), los procesos configuracionales han presentado modificaciones teniendo en cuenta las necesidades de la comunidad de Jongovito en función de la salud pública, la recuperación de espacios físicos, el deterioro ambiental, la fragmentación del sentido de comunidad y apoyo social.

Se evidencia que entre la comunidad, los jóvenes son los que presentan en menor medida el sentimiento de arraigo territorial y mutualidad (Del Pino \& Sánchez, 2008), por cuanto no reconocen la historia de su comunidad, los puntos de encuentro, los proyectos que se están ejecutando, entre otros. Sin embargo, el análisis de esta realidad fomenta la unión de otros grupos (religiosos, institucionales, sociales) y, a su vez, estrechan sus lazos y presentan aspectos en común 
(Maya, 2014). Sin embargo, es necesario considerar que la población adulta mayor e incluso los niños y niñas pueden ser parte de la exclusión a futuro de los miembros de la comunidad ya que sus costumbres y visiones son diferentes a las de la gran mayoría (Delgado, 2006). Por eso, se determina la importancia de la oralidad como fuente transformadora y de reconstrucción histórica que permite disminuir el individualismo y fomenta las acciones colectivas y la identidad comunitaria (Chamorro, 2009).

En cuanto a las dimensiones del apoyo social, se resaltan sus características derivadas de los procesos relacionales vividos antes y durante los procesos de conurbación y ante los cuales la comunidad tiene que hacer frente por medio de sus recursos. En este sentido, se presenta una relación teórica evidente en lo expuesto por Bowling (1991), el cual plantea que el ser humano posee la capacidad para buscar ayuda emocional, instrumental o económica en su red vincular, de ahí que los habitantes han desarrollado diferentes formas de interacción e integración social, por ejemplo, la construcción de organizaciones comunales que lideran los procesos y favorecen la gestión gubernamental, la conservación de estrategias de comunicación y la participación en proyectos sociales. Por otro lado, las fuentes de apoyo social (House, 1981) permiten identificar el conocimiento que poseen los habitantes sobre las dinámicas de interacción en su comunidad; ya que los cambios significativos son evidentes en las fuentes comunitarias y familiares, por lo que las problemáticas como la pérdida del sentido de pertenencia y la ausencia de escenarios o dinámicas de integración entre las generaciones dificulta la interacción social positiva, permitiendo reconocer que son pocos los elementos en común entre ellos.

Por ejemplo, dentro del estudio se reconoce que para los jóvenes existe una fuente de apoyo virtual, ya que se comunican con sus pares y facilita la comunicación, un aspecto importante que se debe considerar al momento de establecer las fuentes de apoyo social que plantean los autores Fachado et al. (2013), puesto que en sus estudios no se reconoce este elemento.

De igual manera, las transformaciones del sector agrícola y el deterioro del suelo producen una crisis de identidad (Chaparro, 1972), puesto que en la actualidad las actividades agrícolas se han afectado por el crecimiento desmedido de plantas procesadoras de ladrillo o "ladrilleras", ante las que la falta de participación comunitaria ha sido ineficaz. Este proceso de expansión urbana es una forma dramática e irreversible de la transformación del uso de la tierra y los recursos ambientales, considerando que durante décadas las ciudades se han ido expandiendo y, de acuerdo con Martínez (2005), casi todos los proyectos de crecimiento de la población mundial durante los próximos treinta años, se concentrarán en zonas urbanas. Además, y según lo planteado por Moreno (2008), la consecuencia directa de la conurbación es el cambio del uso de la tierra y la cubierta vegetal de la región, ya que se inicia con el desarrollo de áreas pavimentadas, dejando a un lado las actividades agrícolas.

Sin embargo, para la comunidad el crecimiento también conlleva a considerar aspectos positivos como la adecuación de redes de acceso y el mejoramiento de 
servicios públicos (Rojas \& Viveros, 2016) por cuanto las condiciones del sector, los proyectos y programas de desarrollo social e infraestructura se han ejecutado de forma positiva y aumentan la generación de microempresas y fuentes de empleo entre sus miembros. Esto determina que las implicaciones negativas de la conurbación se encuentran asociadas a las relaciones sociales, la pérdida de sentido de identidad, los sistemas de valores, las prácticas tradicionales y la cohesión social, componentes esenciales del sentido de comunidad (McMillan \& Chavis, 1986, citados por Maya \& Ramos, 2004).

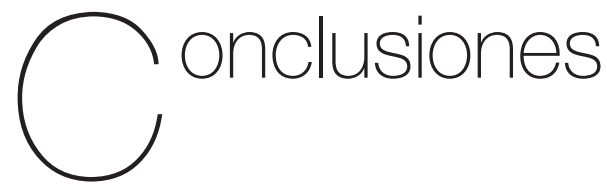

Es importante concluir que el presente estudio cumplió con el objetivo de analizar las implicaciones de la conurbación sobre las dimensiones del sentido de comunidad y el apoyo social en un territorio en situación de conurbación. En este sentido, la conurbación ha presentado diferentes connotaciones; por un lado, el crecimiento en cuanto a su infraestructura, desarrollo de fuentes de empleabilidad, mejoramiento de los servicios públicos y la gestión gubernamental; por otra parte, se evidencia que el crecimiento ha conllevado al desarrollo de problemáticas sociales y ambientales determinantes al momento de construir entornos protectores comunitarios que favorezcan las condiciones de salud mental de los habitantes.

En este sentido, el desconocimiento, la pérdida de identificación con sucesos históricos, lo cambios ambientales, el consumo de sustancias psicoactivas y la seguridad, permiten reconocer que el crecimiento de la ciudad hacia la periferia favorece la instauración de dinámicas que terminan fragmentando los sistemas relacionales de la comunidad, los cuales son el producto de la ausencia de recursos comunitarios y de la pasividad de sus actores para desarrollar planes que mejoren las condiciones.

Los cambios que se logran evidenciar en los componentes del sentido de comunidad repercuten significativamente en las dimensiones del apoyo social, por cuanto los componentes de membresía, influencia y satisfacción de necesidades se encuentran ligados especialmente con el apoyo social efectivo y la interacción social positiva. Además, permiten analizar las necesidades de manera bidireccional, puesto que la disminución o pérdida de los componentes configura las percepciones que se tienen de apoyo social y transforma las fuentes de apoyo social comunes, ya que cada habitante presenta diferentes procesos, de acuerdo a su historia y trayectoria.

Por último, es importante resaltar los esfuerzos que desde la psicología social y comunitaria pueden aportar al análisis de problemáticas tan complejas como la modernización y la conurbación, ya que ponen en evidencia la existencia de consecuencias o repercusiones sociales que afectan la salud mental de los miembros de una comunidad, pero que además da cuenta de los recursos comunitarios que pueden ayudar a combatir situaciones complejas. Además, el interés por rescatar el carácter ético y el compromiso social es indispensable puesto que esta propuesta se basa en un proceso gradual 
que recupera los saberes y brinda relevancia a las expresiones y discursos de sus habitantes.

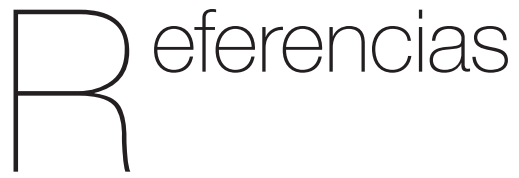

Álvarez, C. (2008). La etnografía como modelo de investigación en educación. Gazeta de Antropología, 24(1-10), 1-15. Recuperado de http://digibug.ugr.es/handle/10481/6998

Bedoya, M., \& Muñoz, L. (2013). Organización y participación comunitaria en el ordenamiento y manejo de los residuos del sector ASOFRUT de la plaza de mercado el Potrerillo en la ciudad de San Juan de Pasto. (Trabajo de grado no publicado). Universidad de Nariño, San Juan de Pasto, Colombia.

Bowling, A. (1991). Social support and social networks: their relationship to the successful and unsuccessful survival of elderly people in the community. An analysis of concepts and a review of the evidence. Pubmed US National Library of Medicine National Institutes of Healt, 1, 6883. Recuperado de https://www.ncbi.nlm. nih.gov/pubmed/2044876

Burbano, A., Narváez, Y., \& Palacios, A. (2017). Evaluación comunitaria en el Corregimiento de Jongovito. (Informe de trabajo no publicado). Universidad de Nariño.

Buss, M., Coelho, S., De Oliveira, I., López, M., \& Mikla, M. (2013). Grupo focal: Una técnica de datos en investigaciones cualitativas. Index de Enfermería, 22(1-2), 75-78.
Recuperado de https://dx.doi.org/10.4321/ S1132-12962013000100016

Bonilla, E., Panadero, S., Rivas, E., Vásquez, J., \& Vásquez, R. (2017). Influencia del apoyo social en el mantenimiento de la convivencia con el agresor en víctimas de violencia de género de León (Nicaragua). Informes Psicológicos, 18(1), 145-165. http://dx.doi.org/10.18566/infpsic.v18n1a08

Buriticá, W., Chaverra, D., \& Monsalve, M. (2015). Caracterización y evaluación de la habilidad de razonabilidad en la producción escrita de textos multimodales. Forma y Función, 28(2), 111-113. Recuperado de http://www.scielo.org.co/pdf/fyf/v28n2/ v28n2a05.pdf

Cerquera, A., Matajira, Y., Salas, M., \& Uribe, A. (2018). Caracterización del dolor osteomuscular y apoyo social percibido en adultos mayores de la ciudad de Bucaramanga, Colombia. Informes Psicológicos, 19(2), 25-41. http://dx.doi. org/10.18566/infpsic.v19n2a02

Chamorro, M. (2013). Sentido de comunidad como una construcción del adolescente "rural" desde los imaginarios alrededor de lo rural-urbano. (Trabajo de grado no publicado). Universidad de Nariño, San Juan de Pasto, Colombia.

Chamorro, G. (2009). Identidad social en el proceso de construcción del plan de vida delMunicipio de el Rosario (Nariño). (Trabajo de grado no publicado). Universidad de Nariño, San Juan de Pasto, Colombia.

Chaparro, P. (1972). Efectos sociales y políticos del proceso de urbanización. Revista EURE Estudios Urbano Regionales, 2(6), 101-113. Recuperado de http://www.eure.cl/index. php/eure/article/view/852/690 
Cueto, R., Espinosa, A., Guillén, H., \& Seminario, M. (2016). Sentido de Comunidad Como Fuente de Bienestar en Poblaciones Socialmente Vulnerables de Lima, Perú. Psykhe. 25(1), 1-18. Recuperado de http:// www.redalyc.org/pdf/967/96745598004. pdf

Da Cunha, J., \& Vignoli, J. (2009). Crecimiento urbano y movilidad en América Latina. Revista Latinoamericana de Población, 3, 27-64. Recuperado de http://www.redalyc. org/articulo.oa?id=323827368003.

Delgado, M. (2006). Percepción social de los vendedores estacionarios de la antigua plazoleta Santander frente al proceso de desarrollo y reubicación de su sitio de trabajo. (Trabajo de grado no publicado). Universidad de Nariño, San Juan de Pasto, Colombia.

Fachado, A. Menéndez, M. \& González, L. (2013). Apoyo social: mecanismos y modelos de influencia sobre la enfermedad crónica. Para saber de, 19, 118-123. Recuperado de http://www.agamfec.com/ wp/wpcontent/uploads/2014/07/19_2_ ParaSaberDe_3.pdf

Fardella, C., Jiménez, F., \& Muñoz, C. (2017). A micro-ethnographic approach to teaching practices in multicultural schools. Tensions and challenges around the schooling of immigrants and minority groups. Perfiles Educativos, 39(156), 71 88. Recuperado de http://www.scielo. org.mx/pdf/peredu/v39n156/0185-2698peredu-39-156-00072.pdf

Gómez, Y., \& Villalobos, F. (2014). Competencias para la formulación de un proyecto de investigación. Guía metodológica para docentes investigadores. Recuperado de http://psicologiaysalud.udenar. edu.co/wp-content/uploads/2016/02/ Competencias-para-la-formulacion-de-unproyecto-de-investigacion.pdf.

House, J. (1981). Work stress and social support. Massachusetts: Addison-Wesley. Recuperado de https://babel.hathitrust. org/cgi/pt?id=mdp.39015071886035

Jociles. M. (2018). La observación participante en el estudio etnográfico de las prácticas sociales. Revista Colombiana de Antropología, 54(1), 121-150. Recuperado de http://www.scielo.org.co/pdf/rcan/ v54n1/0486-6525-rcan-54-01-00121.pdf

Martínez, T. (2015). Una reflexión sobre las periferias metropolitanas de la ciudad de Cali, tomando como referente la literatura y el patrimonio construido. Territorios, 33, 63-68. Recuperado de http://www.scielo. org.co/pdf/terri/n33/n33a04.pdf.

Maya, I. (2014). Sentido de comunidad y potenciación comunitaria. Apuntes de Psicología, 22(2), 187-211. Recuperado de https://www.researchgate.net/ publication/28098336_Sentido_de_ comunidad_y_potenciacion_comunitaria.

Maya, J. \& Ramos, V. (2004). Sentido de comunidad, empoderamiento psicológico y participación ciudadana en trabajadores de organizaciones culturales. Psychosocial Intervention, 23(3), 169-176. Recuperado de $\quad$ https://dx.doi.org/10.1016/j. psi.2014.04.001.

McMillan, B. \& Chavis, D. (1986). Sense of community: a definition and theory. Journal of Community Psychology, 14, 6-23. doi:10.1002/15206629(198601)14:13.0.CO;2-। 
Moreno, C. (2008). La conurbación: rizoma urbano y hecho ambiental complejo. Resumen memorias congreso VII Seminario Nacional de Investigación Urbano - Regional, diversidad y desigualdad en los territorios. Recuperado de http://www.bdigital.unal.edu.co/3363/1/ CIM-CONURBACION.pdf

Montero, M. (2004). Introducción a la psicología comunitaria. Desarrollo, conceptos $y$ procesos. Buenos Aires, Argentina: Editorial Paidós. Recuperado de http://saber.ucv. ve/jspui/bitstream/123456789/4207/1/ montero-introduccion-a-la-psicologiacomunitaria

Quintana, A., \& Montgomery, W. (2006). Metodología de Investigación Científica Cualitativa. Revista Psicología: Tópicos de actualidad, 9(15), 47-84. Recuperado de https://cienciassociales.webcindario.com/ PDF/Cualitativa/Inv_quintana.pdf

Rojas, A., \& Viveros, V. (2016). Análisis multitemporal del crecimiento urbano de Pasto, entre 1989 y 2014 usando SIG. Recuperado de http:// ridum.umanizales.edu.co:8080/ xmlui/bitstream/handle/6789/3039/ VIVEROS_SOTO_VIVIANA_2016. pdf?sequence=1\&isAllowed=y.
Ruano, F., Hernández, M., \& Dorado, A. (2019). La evaluación comunitaria: una herramienta de integración social en los habitantes de la comunidad "Arcoiris". Psicoespacios, 13(22), 40-61. Recuperado de http:// revistas.iue.edu.co/revistasiue/index.php/ Psicoespacios/article/view/1197/1438

Suárez, D. (2015). Nuevos migrantes, viejos racismos: Los mapas parlantes y la niñez migrante en Chile. Revista Latinoamérica de Ciencias Sociales, Niñez y Juventud, 13(2), 627-643. Recuperado de http://www. scielo.org.co/pdf/rlcs/v13n2/v13n2a06.pdf

Del Pino, M., \& Sánchez, L. (2008). Una mirada a la participación comunitaria en el proceso de contraloría social. Paradigma, 29(2), 35-53. Recuperado de ttp://www.scielo. org.ve/scielo.php?script=sci_arttext\&pid $=$ S1011-22512008000200003. 